\title{
Optimal choice and beliefs with ex ante savoring and ex post disappointment ${ }^{1}$
}

\author{
Christian Gollier $^{2} \quad$ Alexander Muermann ${ }^{3}$
}

This version: November 2009

\footnotetext{
${ }^{1}$ We wish to thank Neil Doherty, Howard Kunreuther, Olivia Mitchell, Harris Schlesinger, Stephen Shore, Arthur Snow, Justin Wolfers, and seminar participants at the Wharton School Applied Economics workshop, the Risk Theory Society, the FUR XII, and the EGRIE for their valuable comments. The research leading to these results has received funding from the European Research Council under the European Community's Seventh Framework Programme (FP7/2007-2013) Grant Agreement no. 230589.

${ }^{2}$ Gollier: Institut d'Economie Industrielle, University of Toulouse I, Place Anatole France, 31042 Toulouse cedex, France, email: gollier@cict.fr

${ }^{3}$ Muermann: Department of Finance and Accounting, Vienna University of Economics and Business Administration, Heiligenstaedter Str. 46-48, A-1180 Wien, Austria, email: alexander.muermann@wu-wien.ac.at
} 


\begin{abstract}
We propose a new decision criterion under risk in which people extract both utility from anticipatory feelings ex ante and disutility from disappointment ex post. The decision maker chooses his degree of optimism, given that more optimism raises both the utility of ex ante feelings and the risk of disappointment ex post. We characterize the optimal beliefs and the preferences under risk generated by this mental process and apply this criterion to a simple portfolio choice/insurance problem. We show that these preferences are compatible with first-degree and second-degree stochastic dominance, are consistent with the preference reversal in the Allais paradox, and predict that the decision maker takes on less risk compared to an expected utility maximizer. This speaks to the equity premium puzzle and to the preference for low deductibles in insurance contracts.
\end{abstract}

JEL Classification D81, D84, G11

Keywords endogenous beliefs, anticipatory feeling, disappointment, optimism, decision under risk, portfolio allocation 


\section{Introduction}

In the classical expected utility (EU) model, decision makers are assumed to be stonemen. The risks that they take have no effect on their felicity before the resolution of the uncertainty, which means that they have no anticipatory feelings, no anxiety. Moreover, once the uncertainty is resolved, they evaluate the final outcome in a vacuum. In particular, they feel no disappointment if the final outcome does not attain their expectation. These assumptions are contradicted by introspection. When one of the two authors prepares for a marathon, he faces much uncertainty about his performance on the day of the race. He may form beliefs about it during the threemonth training period. If he is optimistic, he will savor his expected success during that period, but he faces the risk of being disappointed ex post if the outcome is below his expectation. On the contrary, he may prefer to be pessimistic, and thereby depressed during the training period, but with the potential benefit of performing better than expected on the day of the race, yielding much rejoicing ex post. Similarly, suppose the other author forms his beliefs about getting tenure. If he is optimistic about the outcome of the tenure process, he extracts utility from this prospect but faces the risk of being disappointed after the fact. Alternatively, he could be pessimistic and feel miserable, but he is likely to be positively surprised. Similar illustrations can be described in various contexts, from the anxiety generated by a chronic disease to the performance of our private pension account.

In this paper, we take into account of both anticipatory feelings and disappointment. Disappointment theory was first introduced by Bell (1985). Bell observes that the effect of a salary bonus of $\$ 5,000$ on the worker's welfare depends upon whether the worker anticipated no bonus or a bonus of $\$ 10,000$. Bell builds a theory of disappointment on this observation, taking the anticipated payoff as exogenous. However, one difficulty with this theory is that everyone would prefer to have the most pessimistic expectation ex ante, in order to eliminate the risk of disappointment ex post. Thus, Bell's theory is incomplete as a general theory of decision under risk. Gul (1991) provides an axiomatic foundation for preferences that weight outcomes differently above and below an anticipated payoff, which is defined as the certainty equivalent.

In this paper, we explore the idea that people anticipate the future because they extract pleasure from dreaming and savoring the good things that could happen to them. Anticipatory feelings and 
the formation of endogenous beliefs were first introduced in economics by Akerlof and Dickens (1982). In their model, individuals have preferences not only over states of the world but also over their beliefs about the state of the world. Furthermore, individuals have control over their beliefs and select those to maximize their welfare. Beliefs are therefore directly chosen based on preferences over those. Alternatively, Caplin and Leahy (2001) present a model in which individuals make decisions given that they feel anxiety about the future. Thus, in their model, individuals do not directly choose beliefs, but their beliefs are influenced by their own actions. Similarly, Kopczuk and Slemrod (2005) examine the effect of death anxiety on individuals' choice. Brunnermeier and Parker (2005) and Gollier (2005) use a simple portfolio choice model to show how anticipatory feelings can explain why people can rationally be more optimistic than available information suggests they should be. In their model, individuals extract felicity from being optimistic about the future while knowing that an excess of optimism would induce them to take too much risk. As in Akerlof and Dickens (1982), individuals choose their beliefs based on preferences over those. In these studies, individuals are systematically biased in favor of optimism and take on more risk than the EU model predicts, which is counterfactual. A substantial fraction of households do not hold any stocks and the equity premium puzzle would be even more pronounced if individuals were systematically optimistic. Furthermore, in the model of Brunnermeier and Parker (2005) and Gollier (2005) individuals might prefer to have no choice. A reduced choice set limits the cost associated with distorted actions based on distorted beliefs and thereby allows individuals to extract additional felicity from being even more optimistic about the future. In the extreme scenario when there is only one possible action, individuals choose the most optimistic beliefs since actions cannot be distorted at all.

Our model combines Bell's disappointment theory with Akerlof and Dickens' notion of anticipatory feelings. Disappointment is introduced by assuming that the ex post utility is decreasing in the anticipated payoff. The pleasure extracted from anticipatory feelings is measured by the expected future utility based on the subjective beliefs about the distribution of the risk. We establish a link between these subjective beliefs and the anticipated payoff by assuming that the latter equals the subjective certainty equivalent of the risky final payoff. The optimal subjective belief and its corresponding anticipated payoff is thus the best compromise between the willingness to provide pleasure ex ante by being optimistic, and the desire to be pessimistic in order to escape 
disappointment ex post.

There exists experimental evidence about the trade-off between optimism and disappointment that underlies the formation of beliefs in our model. Shepperd and McNulty (1998) and Shepperd and McNulty (2002) provide evidence that people's feeling about an outcome is determined in part by counterfactual alternatives which can come from expectations about the future. In an experiment with recreational basketball players, McGraw et al. (2004) show that overconfidence while making shots is negatively correlated with the average pleasure of the outcome. Moreover, there exists experimental evidence in various settings that individuals abandon optimism or even become pessimistic to avoid disappointment (see e.g. Shepperd et al., 1996, Taylor and Shepperd, 1998, Van Dijk et al., 2003, Carroll et al., 2006). In these studies, it is shown that feedback about outcomes must be both self-relevant and in temporal proximity for individuals to lower their expectations in order to avoid disappointment. In addition, Carroll et al. (2006) argue that bracing to avoid disappointment is more common than bracing to avoid regret.

The idea that a reference point - the anticipated payoff — is endogenously determined relates our paper to Köszegi and Rabin (2006, 2007), who develop a model of reference-dependent preferences and loss aversion. The reference point in their model is defined as the agent's expectations about the distribution of outcomes that are determined by his decision, which in turn is determined by his expectations. The reference point is then determined by the assumption that expectations are fully rational and thus consistent with the implied decision. In our approach, the reference point is endogenously determined through the agent's direct choice of beliefs. These beliefs are the optimal solution that trades off ex ante savoring against ex post disappointment.

The aims of this paper are twofold. In addition to explaining the formation of subjective beliefs, we derive a new decision criterion under risk. Our preference functional is the maximum weighted sum of the subjective expected utility generated from anticipatory feelings and the disappointmentsensitive objective expected utility of the final payoff. We show that this preference functional is compatible with first-degree and second-degree stochastic dominance, but that it does not satisfy the independence axiom. Contrary to Brunnermeier and Parker (2005) and Gollier (2005), it is independent of the set of alternative choices. Therefore, changing the set of choices does not influence the trade-off between savoring and disappointment and thus the value of a specific lottery. Moreover, the preference functional yields a positive value of information even when this information 
has no impact on decisions. We show that it explain the Allais paradox if the individual's degree of absolute risk aversion is increasing in the anticipated payoff. Last, we apply our decision criterion to a simple portfolio/insurance decision problem and show that individuals are more reluctant to take on risk than the EU model predicts and that this reluctance is increasing in the degree of optimism. Our decision criterion thus speaks to the equity premium puzzle and the preference for low deductibles in insurance contracts. Furthermore, it is consistent with Ben Mansour et al. (2008) who apply a Bayesian approach to a lottery survey which comprises data on both risk aversion and subjective beliefs and find a positive correlation between optimism and risk aversion.

The structure of the paper is as follows. In the next section, we describe the preference including the endogenous formation of beliefs. We describe the characteristics of optimal beliefs and choice under these preferences in Section 3 and apply them to a portfolio choice problem in Section 4 . We conclude in Section 5 .

\section{Description of preferences}

Our model has two dates. At date 1, the agent makes a decision under risk. Once the decision has been made, he forms subjective beliefs about the final outcome. These subjective beliefs can differ from the objective probability distribution of the final payoff. He extracts pleasure from savoring this prospect. At date 2, uncertainty is resolved.

We consider a set of lotteries with fixed support $\left\{c_{1}, c_{2}, \ldots, c_{S}\right\}$, where $S$ is the number of possible real-valued lottery payoffs $c_{s}$. Without loss of generality, we assume that $c_{1}<c_{2}<\ldots<c_{S}$. Let $Q$ denote a lottery in this set. It is described by a vector of objective probabilities $Q=\left(q_{1}, \ldots, q_{S}\right)$ in the simplex of $R^{S}$, where $q_{s}$ is the objective probability of lottery payoff $c_{s}$. Let $y$ denote the real-valued anticipated payoff for this lottery. We will formalize below how $y$ is determined by the agent. Once the lottery payoff $c_{s}$ is revealed at date 2 , the agent enjoys a utility $U\left(c_{s}, y\right)$ from the lottery payoff $c_{s}$ given the anticipated payoff $y$.

We suppose that the agent is an expected-utility maximizer in the sense that he evaluates his satisfaction ex ante by $\Sigma_{s} q_{s} U\left(c_{s}, y\right)$. We assume that the bivariate function $U$ is at least twice differentiable. In addition, we assume that, for a given expectation $y$, the agent is averse to risk on the lottery payoff in the sense that the bivariate utility function $U$ is increasing and concave in its 
first argument: $U_{c}>0$ and $U_{c c} \leq 0$. The second variable $y$ in the utility function plays the role of a reference point, from which the agent will evaluate his disappointment or elation. Disappointment is introduced into the model by assuming that $U$ is a decreasing function of the anticipated payoff: $U_{y} \leq 0$. A higher expectation reduces ex post utility at all consumption levels. Your satisfaction of receiving a $\$ 5,000$ salary bonus is larger if you anticipated receiving nothing than if you anticipated receiving $\$ 10,000$.

At date 1 , after having selected lottery $Q=\left(q_{1}, \ldots, q_{S}\right)$, but before its uncertainty is resolved, the agent forms subjective expectations about the distribution of the final payoff. The subjective distribution $P=\left(p_{1}, \ldots, p_{S}\right)$ can differ from the objective one. This means that the agent faces some cognitive dissonance in the decision process. Subjective beliefs play two roles in our model. First, they determine the satisfaction extracted at date 1 from anticipatory feelings. Following Caplin and Leahy (2001) or Brunnermeier and Parker (2005) for example, this level of satisfaction is assumed to be proportional to the subjective expected utility of the future payoff that is measured by $\Sigma_{s} p_{s} U\left(c_{s}, y\right)$. This provides an incentive to be optimistic about the future.

Second, subjective beliefs also determine the level of the anticipated payoff. We assume that the anticipated payoff equals the subjective certainty equivalent of the risk: ${ }^{1}$

$$
U(y, y)=\sum_{s=1}^{S} p_{s} U\left(c_{s}, y\right)
$$

Based on his subjective beliefs, the agent is indifferent between the risky payoff of the selected lottery and the anticipated payoff for sure. One immediate consequence of this definition of the anticipated payoff is that it must be between the smallest possible payoff $c_{1}$ and the largest possible one $c_{S}$. It is intuitive that agents compare ex post the actual payoff of the lottery to its certainty equivalent, and form disappointment or elation from this comparison. We assume that $v(y)=U(y, y)$ is increasing in $y .{ }^{2}$ Intuitively, this means that increasing expectations ex ante raises the satisfaction extracted from anticipatory feelings. This is not guaranteed from our previous assumptions, since an increase in $y$ has an adverse effect on ex post utility. This assumption requires that along the $45^{\circ}$ line, increasing consumption and expectations by the same amount has a positive effect on

\footnotetext{
${ }^{1}$ This assumption is consistent with Gul's (1991) axiomatic theory of disappointment in which outcomes below the objective certainty equivalent are weighted more than those above that threshold.

${ }^{2}$ Notice that $v(c)$ is the utility generated by payoff $c$ when it is perfectly in line with the expectation.
} 
utility. One prefers to consume $\$ 6,000$ in line with expectations, than to consume $\$ 5,000$ in line with expectations.

The agent selects his subjective beliefs and the associated anticipated payoff in order to maximize his intertemporal welfare $W$, which is assumed to be a weighted sum of the satisfaction generated by anticipatory feelings at date 1 and of the satisfaction generated by the final payoff at date 2 :

$$
\begin{gathered}
W(Q)=\max _{P \in \mathcal{S}, y} k \sum_{s=1}^{S} p_{s} U\left(c_{s}, y\right)+\sum_{s=1}^{S} q_{s} U\left(c_{s}, y\right) \\
\text { s.t. } U(y, y)=\sum_{s=1}^{S} p_{s} U\left(c_{s}, y\right) .
\end{gathered}
$$

Parameter $k$ measures the intensity of the decision maker's anticipatory feelings. Observe that, in the process of forming his subjective beliefs, the agent manages some cognitive dissonance. Namely, when computing his intertemporal satisfaction, he is able to take into account the role of his subjective beliefs on his pleasure ex ante, and of the objective distribution of the risk on his pleasure ex post.

The trade-off of the manipulation of beliefs is clear from the definition of the intertemporal welfare function $W$. It is natural to define optimism by the property that $P$ second-degree stochastically dominates $Q$. The selection of an optimistic subjective distribution is thus good for savoring the risk ex ante. However, optimism raises the anticipated payoff $y$, which is bad for satisfaction ex post. In fact, the optimal level of optimism or pessimism is a compromise between two conflicting objectives: 1 . the willingness to savor and dream ex ante which is sustained by optimism, and 2. the desire to escape the risk of disappointment ex post which is sustained by pessimism.

Observe that, by using constraint (3), we can rewrite $W(Q)$ as

$$
W(Q)=\max _{c_{1} \leq y \leq c_{S}} F(y, Q)=k v(y)+\sum_{s=1}^{S} q_{s} U\left(c_{s}, y\right)
$$

$P$ does not explicitly appear in the above program. This is because a sufficient statistic of the ex ante welfare is the anticipated payoff $y$. All subjective beliefs yielding the same certainty equivalent $y$ defined by (3) generates the same total welfare $W$. It implies that our model does not yield a 
unique optimal subjective probability distribution $P$ for each possible objective distribution $Q$. In the remainder of the paper, we focus on characterizing the optimal expectation $y$, which is a scalar. Let $y^{*}=y^{*}(Q)$ denote the anticipated payoff that solves the above program. Any subjective beliefs $P$ that satisfy condition (3) with $y=y^{*}$ will be an optimal subjective distribution. Limiting $y$ to belong to the support $\left[c_{1}, c_{S}\right]$ in program (4) guarantees that there exists a subjective distribution $P$ satisfying condition (3) for the solution of program (4). ${ }^{3}$

Notice that the optimal anticipated payoff $y^{*}(Q)$ and the welfare $W(Q)$ are independent of the set of alternative lotteries. Even when there is only one lottery $Q$, program (4) yields a solution $y^{*}(Q)$ sensibly trading off savoring against disappointment. This is different in the studies by Brunnermeier and Parker (2005) and Gollier (2005) where individuals trade off distorted beliefs against distorted actions. Individuals' welfare then depends on alternative lotteries and might be decreasing in the number of alternative lotteries. When there is only one lottery $Q$, individuals choose the most optimistic beliefs as actions cannot be distorted.

In the following section, we describe the properties of the optimal subjective anticipated payoff $y^{*}(Q)$ and of the preferences functional $W(Q)$.

\section{The optimal anticipated payoff and the preference functional}

\subsection{Characteristics of the optimal anticipated payoff}

At this stage, let us assume that the objective function $F$ in program (4) is concave in the decision variable $y$. Notice that this is not guaranteed by our initial assumptions, since $U$ is not assumed to be concave in its second argument. The first-order condition, which is thus necessary and sufficient, is written as

$$
F_{y}\left(y^{*}, Q\right)=k v^{\prime}\left(y^{*}\right)+\sum_{s=1}^{S} q_{s} U_{y}\left(c_{s}, y^{*}\right)\left\{\begin{array}{l}
\leq 0 \quad \text { if } \quad y^{*}=c_{1} \\
=0 \quad \text { if } \quad y^{*} \in\left[c_{1}, c_{S}\right] \\
\geq 0 \quad \text { if } \quad y^{*}=c_{S}
\end{array}\right.
$$

Parameter $k$ depends upon both psychological and contextual elements. People who are more

\footnotetext{
${ }^{3}$ We note that the fact that our model does not pin down a unique optimal subjective belief $P$ is not problematic for the definition of optimism. Any subjective beliefs $P^{1}$ that second-degree stochastically dominate beliefs $P^{2}$ imply a higher optimal anticipated payoff $y^{1} \geq y^{2}$.
} 
sensitive to anticipatory feelings have a larger $k$. If the duration of the period separating the decision and the resolution of the uncertainty is increased, people have more time to savor their dream, which also implies a larger $k$. It is interesting to examine the effect of an increase in $k$ on the optimal anticipated payoff. When $k=0$, the agent has no anticipatory feelings. Because the ex post utility is decreasing in the anticipated payoff, it is optimal to set it to its minimum $y_{k=0}^{*}(Q)=c_{1}$ for all $Q$. In that case, $W(Q)=\Sigma_{s} q_{s} U\left(c_{s}, c_{1}\right)$, which implies that $W$ is an expectedutility functional with utility function $u(c)=U\left(c, c_{1}\right)$. On the contrary, when $k$ tends to infinity, only anticipatory feelings matter. In that case, it is optimal to set $y$ to its maximum level, since the welfare $v$ extracted from anticipatory feelings is increasing in the anticipated payoff. Thus, $y_{k=+\infty}^{*}(Q)=c_{S}$ for all $Q$. Here also, $W(Q)$ is an expected utility functional with a utility function $u(c)=U\left(c, c_{S}\right)$. In the following proposition, we show that $y^{*}$ is increasing in the intensity $k$ of anticipatory feelings.

Proposition 1 An increase in the intensity of anticipatory feelings weakly increases the optimal anticipated payoff.

Proof. Because we assume that $F_{y y}$ is negative, the sign of $d y^{*} / d k$ is the same as the sign of $F_{y k}$. Since $F_{y k}=v^{\prime}\left(y^{*}\right)>0$, we obtain the result.

When the intensity of anticipatory feelings increases, people get more benefits from their dream. This provides more incentive to distort their beliefs in favor of optimism. As mentioned above, the parameter $k$ can be interpreted as a measure for the duration of the period between the decision and the resolution of uncertainty. The proposition implies that individuals would lower their optimal anticipated payoff as the resolution of uncertainty approaches. This prediction is consistent with the experimental evidence in different settings that individuals lower their expectations to avoid disappointment if the feedback about outcomes is in temporal proximity (see e.g. Shepperd et al., 1996, Taylor and Shepperd, 1998, Van Dijk et al., 2003, Carroll et al., 2006).

Next, we examine how the optimal expectations are influenced by the objective probability distribution, i.e., we examine the characteristics of the function $y^{*}(Q)$. The intuition suggests that a deterioration in the objective risk should reduce the optimal expectation. In the following two propositions, we investigate two types of changes in the objective risk: first-degree stochastic dominance (FSD) and Rothschild-Stiglitz increases in risk (IR). We determine the condition for 
each type of changes in risk under which a change in risk reduces (resp. increases) the optimal expectation.

Proposition 2 Any change that deteriorates the objective risk in the sense of first-degree stochastic dominance weakly reduces (resp. increases) the optimal expectation $y^{*}$ if $U_{y}$ is increasing (resp. decreasing) in the payoff $c$.

Proof. Consider two distributions, $Q^{a}$ and $Q^{b}$, with $Q^{b}$ being FSD-dominated by $Q^{a}$. Let $y^{a}$ and $y^{b}$ denote the optimal anticipated payoff respectively under objective probabilities $Q^{a}$ and $Q^{b}$. Assume that $y^{a}$ is an interior solution, so that

$$
F_{y}\left(y^{a}, Q^{a}\right)=k v^{\prime}\left(y^{a}\right)+\sum_{s=1}^{S} q_{s}^{a} U_{y}\left(c_{s}, y^{a}\right)=0
$$

Suppose that $U_{y}$ is increasing in $c$. By definition of the FSD order, this implies that $F_{y}\left(y^{a}, Q^{b}\right) \leq$ $F_{y}\left(y^{a}, Q^{a}\right)=0$. Because $F_{y y}$ is assumed to be negative, this implies that $y^{b}$ must be smaller than $y^{a}$. It is easy to extend this proof for corner solutions by using the Kuhn-Tucker conditions (5).

The intuition suggests that a FSD deterioration in the objective distribution should reduce the optimal anticipated payoff. This is true only if $U$ is supermodular, i.e., if the marginal utility of consumption is increasing in the anticipated payoff $y$. Increasing the payoff from $\$ 5,000$ to $\$ 6,000$ has a smaller effect on satisfaction if one anticipated receiving nothing than if one anticipated receiving $\$ 10,000$. This property of $U$ induces the decision-maker to be correlation-loving in the terminology of Eeckhoudt, Rey and Schlesinger (2007). In our context in which the second argument of the utility function is interpreted as the anticipated payoff, it is intuitive to make this assumption. Following the above-mentioned authors, let us consider the following thought experiment in which anticipated payoffs are allowed to be state-contingent. One is offered to choose between two lotteries entailing two payoffs $c_{-}$and $c_{+}>c_{-}$, and two anticipated payoffs $y_{-}$and $y_{+}>y_{-}$. In lottery A, one gets either $\left(c_{-}, y_{-}\right)$or $\left(c_{+}, y_{+}\right)$with equal probabilities. In lottery $\mathrm{B}$, one gets either $\left(c_{-}, y_{+}\right)$ or $\left(c_{+}, y_{-}\right)$with equal probabilities. Condition $U_{c y}>0$ implies that the expected utility of $\mathrm{B}$ is smaller than the expected utility of $\mathrm{A}$, in which the anticipated payoffs are more in line with actual payoffs. This thought experiment illustrates the fact that whereas condition $U_{y}<0$ is a notion of disappointment, condition $U_{c y}>0$ is a notion of disappointment aversion. Disappointment 
aversion is required here to guarantee that any deterioration of the objective risk in the sense of FSD deteriorates the optimal anticipated payoff. It implies that it is never the case that the optimal subjective beliefs $P$ improve in the sense of FSD when the objective beliefs are deteriorating in the same sense.

In the following proposition, we examine the effect of an increase in the objective risk on the optimal anticipated payoff.

Proposition 3 Any increase in the objective risk in the sense of Rothschild-Stiglitz weakly reduces (resp. raises) the optimal expectation $y^{*}$ if $U_{y}$ is concave (resp. convex) in the actual payoff.

Proof. The proof of this proposition is parallel to the proof of Proposition 2. It is based on the fact that $\Sigma_{s} q_{s} U_{y}\left(c_{s}, y^{*}\right)$ is reduced by an increase in risk if $U_{y}$ is concave in $c$.

The effect of an increase in risk on $y^{*}$ is more problematic than in the case of FSD, since its sign depends upon whether $U_{c c y}$ is positive or negative. We believe that $U_{y}$ should be concave in $c$. To show this, let us again use the technique introduced by Eeckhoudt, Rey and Schlesinger (2007) by considering two lotteries involving now one sure payoff $c$ and another risky one $c+\widetilde{\varepsilon}$, with $E \widetilde{\varepsilon}=0$. Lottery A offers either $\left(c+\widetilde{\varepsilon}, y_{-}\right)$or $\left(c, y_{+}\right)$with equal probabilities. In lottery $\mathrm{B}$, expectations are reversed, with outcomes $\left(c+\widetilde{\varepsilon}, y_{+}\right)$and $\left(c, y_{-}\right)$. Condition $U_{c c y} \leq 0$ guarantees that lottery B yields a smaller expected utility than lottery A.

\subsection{Characteristics of the preference functional}

We observe that program (4) is relevant to determine the optimal lottery choice at date 1 . It thus provides a new decision criterion under risk. To get more insights on the characteristics of the preference functional $W$ that this model generates, let us rewrite $W$ as follows:

$$
W\left(q_{1}, \ldots, q_{S}\right)=\sum_{s=1}^{S} q_{s} M\left(c_{s}, y^{*}(Q)\right)
$$

where $y^{*}(Q) \in \arg \max _{y} \sum_{s=1}^{S} q_{s} M\left(c_{s}, y\right)$ and the functional $M$ is defined as

$$
M(c, y)=k v(y)+U(c, y) .
$$

We notice that this representation is a special case of the "induced preference functional over 
temporal risk" (see Kreps and Porteus (1979), Machina (1984)) where the anticipated payoff $y$ is the intermediate or "auxiliary" choice variable which is to be determined before the uncertainty of the lottery $Q$ is resolved. Machina (1984) applied the technique used in his Generalized Expected Utility (GEU) model (Machina (1982)). Within the GEU model, the functional $M$ is what Machina defines as the "local utility function." The results regarding preferences over temporal prospects thus apply to our model. ${ }^{4}$

In contrast to postulating the existence of an "auxiliary" choice variable $y$ and a smooth local utility function $M$, we derive both as a rational mental process based on both anxiety and fear of disappointment, whose impact on satisfaction is measured by a von Neumann-Morgenstern expected utility functional. Notice also that our model is in a sense simpler than Machina's GEU model, since our local utility function depends upon distribution $Q$ only through the one-dimensional anticipated payoff $y^{*}(Q)$.

\subsubsection{Expected-utility functional}

Because the shape of $M$ with respect to $c$ will in general depend upon the objective distribution $Q$, our preference functional does not satisfy the independence axiom. However, there exist some limit cases in which $W$ is additive, so that individuals behave as expected-utility maximizers.

Proposition 4 The following statements are equivalent.

1. W is an expected-utility functional.

2. $U(c, y)=u(c) f(y)+g(y)$ for all $(c, y)$.

3. $d / d y\left(-U_{c c}(c, y) / U_{c}(c, y)\right)=0$ for all $(c, y)$.

Proof. It is easy to check that statements 2. and 3. are equivalent. If $U(c, y)=u(c) f(y)+g(y)$, then $W$ is an expected-utility functional (see Proposition 5, Kreps and Porteus, 1979). Last, if $W$ is an expected-utility functional then $W(Q)=\Sigma_{s} q_{s} M\left(c_{s}, y^{*}(Q)\right)=\Sigma_{s} q_{s} V\left(c_{s}\right)$ for some function $V$ and for all $Q$. Therefore, $V\left(c_{s}\right)=M\left(c_{s}, y^{*}(Q)\right)$ for all $Q$ and $d / d y\left(-M_{c c}(c, y) / M_{c}(c, y)\right)=$ $d / d y\left(-U_{c c}(c, y) / U_{c}(c, y)\right)=d / d y\left(-V_{c c}(c) / V_{c}(c)\right)=0$.

\footnotetext{
${ }^{4}$ We note that the general assumptions in Machina (1984, p. 206) are satisfied in our model.
} 
Two examples of the structural form in statement 2. are $U(c, y)=u(c-\eta y)$ with $u(c)=$ $-\exp (-\alpha c)$ and $U(c, y)=u\left(c y^{-\eta}\right)$ with $u(c)=c^{1-\gamma} / 1-\gamma$. Both examples can be interpreted as habit formation models, the first additive with $u$ exhibiting constant absolute risk aversion, the latter multiplicative with $u$ exhibiting constant relative risk aversion.

\subsubsection{Stochastic dominance}

With the exception of the functional form of $U(c, y)$ specified in Proposition 4, statement $2 ., W$ cannot be represented as an expected-utility functional. However, in the following proposition we show that the preference functional $W$ satisfies the minimal requirement of second-degree stochastic dominance. Remember that second-degree stochastic dominance has first-degree stochastic dominance and Rothschild-Stiglitz increase in risk as particular cases. ${ }^{5}$

Proposition 5 Any second-degree stochastically dominated shift in the objective distribution $Q$ weakly reduces the agent's intertemporal welfare $W$.

Proof. See Theorem 6 in Machina (1984) for a proof in the context of induced preferences over temporal prospects. A simpler proof can be given for our specification. Consider two objective distributions $Q^{a}$ and $Q^{b}$ such that $Q^{b}$ is dominated by $Q^{a}$ in the sense of second-degree stochastic dominance. We have to prove that $W\left(Q^{b}\right)$ is weakly smaller than $W\left(Q^{a}\right)$. Because we assume that $U$ is increasing and concave in its first argument, this implies that

$$
\sum_{s=1}^{S} q_{s}^{b} U\left(c_{s}, y\right) \leq \sum_{s=1}^{S} q_{s}^{a} U\left(c_{s}, y\right)
$$

for all $y$. Applying this for $y^{b}=y^{*}\left(Q^{b}\right)$, we obtain that

$$
\begin{aligned}
W\left(Q^{b}\right) & =k v\left(y^{b}\right)+\sum_{s=1}^{S} q_{s}^{b} U\left(c_{s}, y^{b}\right) \\
& \leq k v\left(y^{b}\right)+\sum_{s=1}^{S} q_{s}^{a} U\left(c_{s}, y^{b}\right) \\
& \leq k v\left(y^{a}\right)+\sum_{s=1}^{S} q_{s}^{a} U\left(c_{s}, y^{a}\right) \\
& =W\left(Q^{a}\right) .
\end{aligned}
$$

\footnotetext{
${ }^{5}$ Notice that this proposition would not necessarily hold if the constraints on $y$ would depend on the characteristics of the objective distribution $Q$.
} 
The first inequality is condition (8) applied for $y=y^{b}$, whereas the second inequality comes from the fact that $y^{a}$ is the optimal anticipated payoff for objective risk $Q^{a}$.

This proposition means that optimizing subjective beliefs can never compensate for a deterioration of the objective risk.

\subsubsection{Value of Information}

Because the preference functional $W$ is the upper envelop of linear functions of $Q$, it is convex in $Q .{ }^{6}$ This implies that our framework yields a positive value of information even when this information has no impact on decisions. Remember that in the EU model, information has a positive value only if it can be used to reoptimize behavior. In contrast, information is valuable in our framework because of its effect on subjective beliefs. Information allows for a more precise anticipated payoff, which improves the compromise between ex ante savoring and ex post disappointment. To illustrate this point, compare the following two contexts. In the uninformative context, there is a single objective probability distribution $Q$. In the informative context, the decision maker observes a signal $\theta$ prior to selecting his beliefs. Suppose that there are $n$ possible signals $\theta=1, \ldots, n$, each one occurring with probability $\pi_{\theta} \geq 0$, with $\Sigma_{\theta} \pi_{\theta}=1$, and yielding a posterior distribution $Q_{\theta}$, with $\Sigma_{\theta} \pi_{\theta} Q_{\theta}=Q$. The convexity of the preference functional $W$ implies that $\Sigma_{\theta} \pi_{\theta} W\left(Q_{\theta}\right)$ is larger than $W\left(\Sigma_{\theta} \pi_{\theta} Q_{\theta}\right)=W(Q)$. It is strictly larger if there are at least two signals yielding different optimal beliefs.

\subsubsection{Allais paradox}

As shown in Proposition 4, when the anticipated payoff enters into the utility function in a multiplicative way, the principle that beliefs are determined as a best compromise between ex ante savoring and ex post disappointment cannot solve the well-known paradoxes affecting the expected utility model. This is the case when the absolute aversion to consumption risk $-U_{c c}(c, y) / U_{c}(c, y)$ is independent of $y$. Machina $(1982,1987)$ showed how the GEU model can solve the Allais paradox, which is often referred to as the "fanning out" of indifference curves in the Marschak-Machina triangle. In Machina (1982, Theorem 5), it is shown that solving the paradox requires that any FSD-dominated shift in distribution $Q$ reduces the Arrow-Pratt risk aversion of the local utility

\footnotetext{
${ }^{6}$ See Theorem 1, Machina (1984) for the identical result regarding induced preferences over temporal risks.
} 
function $M$, which is measured by $-M_{c c}(c, Q) / M_{c}(c, Q)$, for all $c$ and for all $Q \in \mathcal{S}$.

Proposition 6 Suppose that the decision maker is disappointment-averse, i.e., $U_{c y}$ is nonnegative. The preference functional $W$ fans out - and can thereby explain the Allais paradox - if and only if the absolute aversion to the objective risk is increasing in the anticipated payoff, i.e., if

$$
\frac{\partial}{\partial y}\left(-\frac{U_{c c}(c, y)}{U_{c}(c, y)}\right) \geq 0
$$

for all $(c, y)$.

Proof. This is a consequence of Machina (1982, Theorem 5) and of the fact that the local risk aversion of $M$ is measured by

$$
-\frac{\partial M_{c c}(c, Q)}{\partial M_{c}(c, Q)}=-\frac{U_{c c}\left(c, y^{*}(Q)\right)}{U_{c}\left(c, y^{*}(Q)\right)}
$$

From Proposition 2, we know that $y^{*}$ is reduced by any FSD-deterioration in the objective risk $Q$. This implies that the local risk aversion is reduced by such a change in $Q$ if condition (9) is satisfied.

Thus, the Allais paradox can be explained by our model if any exogenous increase in the anticipated payoff raises risk aversion. Notice that this condition requires that $U_{y}$ be concave in $c$, a condition that we have identified in Proposition 3 as necessary and sufficient for any increase in the objective risk to reduce the optimal anticipated payoff. Moreover, Machina $(1982,1984)$ shows that the "fanning out" of indifference curves can additionally explain the "oversensitivity to changes in the probabilities of small probability-outlying events" and the "utility evaluation effect."

Let us illustrate how this model can solve the Allais paradox by setting $U(c, y)=(1+c-$ $\eta y)^{1-\gamma} /(1-\gamma)$, with $\eta=1 / 2$. Observe that $U_{c}>0, U_{c c}<0, U_{y}<0$, and $U_{c y}>0$. Notice also that $-U_{c c}(c, y) / U_{c}(c, y)=\gamma /(1+c-\eta y)$ is increasing in $y$, so that all conditions in the above proposition are satisfied. We also assume that $\gamma=4$, a number that belongs to the range of risk aversion that most economists believe is reasonable, and that $k=1$, which means that the agent 
weights ex ante and ex post satisfactions equally when measuring his intertemporal welfare.

\begin{tabular}{|cccc|}
\hline Lottery & $Q$ & $y^{*}(Q)$ & $W(Q)$ \\
\hline \hline & $q_{1}=0$ & & \\
$a_{1}$ & $q_{2}=1$ & 1.0000 & -0.1957 \\
& $q_{3}=0$ & & \\
\hline & $q_{1}=0.01$ & & \\
$a_{2}$ & $q_{2}=0.89$ & 0.8574 & -0.2088 \\
& $q_{3}=0.1$ & & \\
\hline \hline & $q_{1}=0.9$ & & \\
$a_{3}$ & $q_{2}=0$ & 0.0263 & -0.6328 \\
& $q_{3}=0.1$ & & \\
\hline & $q_{1}=0.89$ & & \\
$a_{4}$ & $q_{2}=0.11$ & 0.0273 & -0.6339 \\
& $q_{3}=0$ & & \\
\hline
\end{tabular}

Table 1: The optimal anticipated payoff and the intertemporal welfare for Allais' common consequence effect, with $k=1$ and $U(c, y)=-(1+c-y / 2)^{-3} / 3$.

The first Allais' paradox, the common consequence effect, is about two choice problems concerning four lotteries, $a_{1}, a_{2}, a_{3}$ and $a_{4}$, and three possible payoffs, $c_{1}=0, c_{2}=1$ and $c_{3}=5$. Lottery $a_{1}$ is a sure gain of $c=1$. It is easy to check that the optimal anticipated payoff is $y_{1}^{*}=1$ if this lottery is selected, yielding $W_{1}=-0.1975$. The other lotteries, their optimal anticipated payoff, and the resulting intertemporal welfare are summarized in Table 1 . The prediction of the EU model is that if $a_{1}$ is preferred to $a_{2}$, then it must be that $a_{4}$ is preferred to $a_{3}$. This is not the case in our model, since $a_{1}$ is indeed preferred to $a_{2}$, but $a_{3}$ is preferred to $a_{4}$.

The intuition for why our model can explain the Allais' paradox is quite simple. The preference of $a_{1}$ over $a_{2}$ indicates a high degree of risk aversion, whereas the preference of $a_{3}$ over $a_{4}$ indicates a smaller one. This reduction in risk aversion in the second choice context is explained by the fact that it is much less favorable to the agent than in the first choice context. This induces the agent to optimally reduce his expectations, from $y^{*}$ around 1 to $y^{*}$ around 0 . We then get the observed 
preference reversal by observing that our additive habit specification implies a reduction in the agent's risk aversion when his expectations fall.

\subsection{Some specifications}

Let us examine the most standard specifications of $U$ in more detail to see whether the assumptions made in this section are satisfied in the existing literature.

\subsubsection{Bell (1985)}

Bell (1985) considered the following specification

$$
U(c, y)=u(c)+\eta g(u(c)-u(y)),
$$

with $u$ and $g$ being two increasing and concave functions, and $\eta$ being a positive scalar. In this case, $u(c)-u(y)$ measures the intensity of elation. When it is negative, its absolute value measures the intensity of disappointment. The psychological satisfaction associated with elation is an increasing and concave function $\eta g($.$) of its intensity. Because U_{c y}=-\eta g^{\prime \prime}(\Delta u) u^{\prime}(c) u^{\prime}(y)$, this specification exhibits disappointment aversion due to the concavity of function $g$. We also see from this that $U_{c c y}$ is negative if $g^{\prime \prime \prime}$ is positive. Finally, we can check that

$$
-\frac{U_{c c}(c, y)}{U_{c}(c, y)}=-\frac{u^{\prime \prime}(c)}{u^{\prime}(c)}-\frac{\eta g^{\prime \prime}(\Delta u) u^{\prime}(c)}{1+\eta g^{\prime}(\Delta u)} .
$$

This is increasing in $y$ if $g^{\prime \prime \prime}$ is positive. The standard specifications used for $g$ are exponential, power or log functions, whose derivatives alternate in sign $\left(g^{\prime}>0, g^{\prime \prime}<0, g^{\prime \prime \prime}>0\right)$. Thus, Bell's specification is compatible with the properties that the optimal anticipated payoff deteriorates or improves in parallel with the objective distribution of the initial risk, and with the fanning-out hypothesis that can explain Allais' paradoxes. 


\subsubsection{Additive habits}

As already encountered after Proposition 4, another specification is based on the model of additive habits with

$$
U(c, y)=u(c-\eta y)
$$

with $u$ being increasing and concave, and $\eta$ being a positive scalar. ${ }^{7}$ In this case, a unit increase in expectation $y$ has an impact on final utility that is equivalent to a $\eta$ reduction in the actual payoff. This specification is similar to the idea of consumption habit formation developed by Constantinides (1990) in which a unit increase in the level of past consumption habit has an impact on current utility equivalent to a $\eta$ reduction in consumption. Exactly as habits "eat" some of the current consumption in Constantinides' model, expectations "eat" some of the final payoff in this specification of our model. It is immediate that $U_{c y}=-\eta u^{\prime \prime}(c-\eta y)$ is positive, which implies disappointment aversion. Observe in turn that $U_{c c y}$ is negative if $u^{\prime \prime \prime}$ is positive, a standard assumption that is referred to as prudence (Kimball (1990)). Finally, $-U_{c c} / U_{c}$ is increasing in $y$ if $u$ exhibits decreasing absolute risk aversion, a standard assumption in decision theory. Thus, the additive habit specification also implies that the optimal anticipated payoff deteriorates in parallel with the objective risk, and that the $W$ functional fans out, thereby potentially explaining the Allais paradox.

We can get additional properties in this case by using the first-order condition, which can be written as follows under this specification:

$$
k(1-\eta) u^{\prime}\left((1-\eta) y^{*}\right)=\eta E u^{\prime}\left(\widetilde{c}-\eta y^{*}\right)
$$

where $\widetilde{c}$ is the random variable distributed as $Q$. Notice that the second-order condition is automatically satisfied. The following proposition describes some basic properties of the optimal expectations.

Proposition 7 Suppose that $U(c, y)=u(c-\eta y)$. The optimal anticipated payoff satisfies the following properties:

\footnotetext{
${ }^{7}$ A similar approach is the multiplicative habit specification with $U(c, y)=u\left(c y^{-\eta}\right)$, with $u$ being increasing and concave, and $\eta$ being a scalar belonging to interval $[0,1]$. Parameter $\eta$ represents here the percentage reduction in the actual payoff that has an effect on utility equivalent to a one percent increase in the anticipated payoff.
} 
1. $y^{*}$ is smaller than the expected payoff $\mu=\Sigma_{s} q_{s} c_{s}$ if $k$ is smaller or equal to $\eta /(1-\eta)$ and $u^{\prime}$ is convex.

2. Suppose that $\widetilde{c}=\mu+\widetilde{\varepsilon}$ and that $k=\eta /(1-\eta)$. Then $d y^{*} / d \mu$ is larger than unity if $u$ is standard, i.e., if $A(z)=-u^{\prime \prime}(z) / u^{\prime}(z)$ and $P(z)=-u^{\prime \prime \prime}(z) / u^{\prime \prime}(z)$ are two nonincreasing functions.

\section{Proof. See Appendix A.1.}

When $k$ equals $\eta /(1-\eta)$, the optimal anticipated payoff is equal to the sure payoff if there is no objective uncertainty, and is smaller than the objective expected payoff when the outcome is risky and $u$ is prudent. When all payoffs are increased by $\$ 1$, the optimal anticipated payoff is increased by more than $\$ 1$ if $u$ is standard. ${ }^{8}$

An analytical characterization of $W$ can be obtained in the special case in which $u$ is assumed to be quadratic. ${ }^{9}$ Suppose that $u(z)=z-0.5 \beta z^{2}$ for all $z$ in the relevant domain $z \leq 1 / \beta$. It is easy to check that the optimal anticipated payoff $y^{*}(Q)$ equals

$$
y^{*}(Q)=\frac{a}{d}+\frac{b}{d} \sum_{s=1}^{S} q_{s} c_{s}
$$

with $a=k(1-\eta)-\eta, b=\eta \beta$ and $d=k(1-\eta)^{2} \beta+\eta^{2} \beta$. Thus, the optimal anticipated payoff is linear increasing in the expected payoff. We can then rewrite $W(Q)=F\left(y^{*}(Q), Q\right)$ as follows:

$$
W(Q)=\sum_{s=1}^{S} q_{s} u\left(c_{s}\right)+\frac{1}{2} \frac{\left(a+b \sum_{s=1}^{S} q_{s} c_{s}\right)^{2}}{d} .
$$

$W$ is a linear combination of an expected utility functional and of a (convex) quadratic function of the objective expected payoff.

\section{Optimal decision making}

In this section, we examine optimal decision-making under uncertainty with preferences as specified in Section 2. We are particularly interested in the impact of anticipatory feelings and disappoint-

\footnotetext{
${ }^{8}$ To illustrate, the power functions and the logarithmic function are standard.

${ }^{9}$ If $u$ exibits constant absolute risk aversion, then $W$ is an expected-utility functional (see Proposition 4.
} 
ment relative to EU preferences. For this purpose, we present two settings: portfolio choice and insurance demand.

\subsection{Portfolio choice problem}

We investigate the standard one-safe-one-risky-asset model. The agent has some initial wealth $z_{0}$ that can be invested in a safe asset whose return is normalized to zero and in a risky asset whose excess return is described by random variable $\widetilde{x}$. The agent must determine his dollar investment $\alpha$ in the risky asset. He selects $\alpha$, which maximizes his intertemporal welfare $W(\alpha)$ defined as

$$
W(\alpha)=\max _{y_{\min } \leq y \leq y_{\max }} k v(y)+E U\left(z_{0}+\alpha \widetilde{x}, y\right),
$$

where $y_{\min }$ and $y_{\max }$ are the exogenously given minimum and maximum possible expectations. We can solve this problem for each $\alpha$, thereby yielding the optimal anticipated payoff $y(\alpha)$ as a function of the demand for the risky asset. It satisfies the following condition:

$$
k v^{\prime}(y(\alpha))+E U_{y}\left(z_{0}+\alpha \widetilde{x}, y(\alpha)\right)\left\{\begin{array}{lll}
\leq 0 & \text { if } \quad y(\alpha)=y_{\min } \\
=0 & \text { if } \quad y(\alpha) \in\left[y_{\min }, y_{\max }\right] \\
\geq 0 & \text { if } \quad y(\alpha)=y_{\max } .
\end{array}\right.
$$

We assume that $W$ is concave in $\alpha$. By the envelope theorem, the first-order condition for the portfolio problem is written as

$$
W^{\prime}\left(\alpha^{*}\right)=E \widetilde{x} U_{c}\left(z_{0}+\alpha^{*} \widetilde{x}, y^{*}\right)=0
$$

where $y^{*}=y\left(\alpha^{*}\right)$. Because the utility function $U$ is concave in the final payoff, we directly obtain the following result.

Proposition 8 The demand for the risky asset is positive (zero, negative) if the expected excess return is positive (zero, negative). 
Proof. Because we assume that $W$ is concave in $\alpha$, the optimal $\alpha^{*}$ is positive (zero, negative) if $W^{\prime}(0)$ is positive (zero, negative). But we have that

$$
W^{\prime}(0)=E \widetilde{x} U_{c}\left(z_{0}, y(0)\right)=U_{c}\left(z_{0}, y(0)\right) E \widetilde{x} .
$$

Because $U_{c}$ is positive, we can conclude that the sign of $\alpha^{*}$ and of $E \widetilde{x}$ must coincide.

Because our model yields a smooth local utility function that is concave in the final payoff, it exhibits second-order risk aversion as in the standard EU model. Proposition 8 confirms this point.

We now analyze comparative statics for the additive habit specification $U(c, y)=u(c-\eta y)$ for an increasing and concave function $u$ and a positive scalar $\eta<1$. The following proposition describes the effect that changes in the intensity of anticipatory feelings, $k$, and of disappointment, $\eta$, have on the portfolio allocation of the decision maker.

Proposition 9 Suppose that $U(c, y)=u(c-\eta y)$ and $u$ is DARA.

1. The allocation in the risky asset is decreasing in the intensity $k$ of anticipatory feelings.

2. The allocation in the risky asset is decreasing in (increasing in, independent of) $\eta$ if relative risk aversion is uniformly larger than (smaller than, equal to) unity.

\section{Proof. See Appendix A.2.}

For the additive habit specification, DARA is equivalent to absolute risk aversion being increasing in the anticipated payoff, see (9). An increase in the intensity of anticipatory feelings raises the anticipated payoff and thereby increases the degree of risk aversion. This explains the somewhat surprising result that the individual with anticipatory feelings forms a less risky portfolio and that optimism is negatively related to the investment in the risky asset. This prediction is consistent with the empirical result of Ben Mansour et al. (2008) who apply a Bayesian approach to a lottery survey which comprises data on both risk aversion and subjective beliefs and find a positive correlation between optimism and risk aversion. Increasing the intensity of ex-post disappointment has two opposing effects. First, it increases the degree of risk aversion, as $\partial / \partial y\left(-U_{c c}(c, y) / U_{c}(c, y)\right) \geq 0$. Second, it decreases the anticipated payoff and thereby reduces the degree of risk aversion. We have shown that if relative risk aversion is larger than 1 then the first effect dominates the second. 
Under these conditions, individuals with anticipatory feeling and ex-post disappointment select a portfolio that is less risky compared to the traditional EU model. Both psychological phenomena therefore speak to the equity premium puzzle. ${ }^{10}$ Note that this effect applies to both optimistic and pessimistic individuals. This stands in contrast to the literature on optimal expectations (Brunnermeier and Parker, 2005, and Gollier, 2005) in which individuals are always optimistic and select a riskier portfolio, reinforcing the equity premium puzzle.

An analytical solution can be obtained to this problem in the special case with a power utility function under the additive habit specification. Suppose accordingly that $U(c, y)=(1-\gamma)^{-1}(c-$ $\eta y)^{1-\gamma}$. The first-order condition (13) of the portfolio problem is solved in that case by $\alpha^{*}=$ $a\left(z_{0}-\eta y^{*}\right)$ where $a$ is a scalar that solves equation $E \widetilde{x}(1+a \widetilde{x})^{-\gamma}=0$. Given this, the first-order condition (12) of the anticipated payoff $y^{*}$ can be simplified as

$$
k(1-\eta)^{1-\gamma} y^{*-\gamma}=\eta\left(z_{0}-\eta y^{*}\right)^{-\gamma} E(1+a \widetilde{x})^{-\gamma} .
$$

The optimal anticipated payoff is thus equal to

$$
y^{*}=\frac{z_{0}}{\eta+\left[\frac{\eta E(1+a \widetilde{x})^{-\gamma}}{k(1-\eta)^{1-\gamma}}\right]^{1 / \gamma}} .
$$

Observe that $y^{*}$ vanishes when there is no anticipatory feelings $(k=0)$, and that it is increasing in $k$. Notice also that it is proportional to wealth $z_{0}$. From this, we obtain that

$$
\alpha^{*}=\frac{a z_{0}}{1+k^{1 / \gamma}\left(\frac{\eta}{1-\eta}\right)^{\frac{\gamma-1}{\gamma}}\left(E(1+a \widetilde{x})^{-\gamma}\right)^{-1 / \gamma}} .
$$

As in the EU/CRRA case, the share of wealth invested in the risky asset is constant. In the absence of anticipatory feelings, this share is $a$ independent of disappointment aversion $\eta$. The signs of the effect of $k$ and $\eta$ are as demonstrated in Proposition 9.

\footnotetext{
${ }^{10}$ Ang et al. (2005) apply Gul's (1991) disappointment preferences to a portfolio choice problem and show that individuals who are averse to disappointment hold significantly less equity. Our result predicts that individuals who additionally have anticipatory feelings will hold even less equity.
} 


\subsection{Demand for insurance}

In this section, we apply our decision criterion to an insurance purchase decision. The agent is endowed with initial wealth $z_{0}$ and is facing a loss of random size $\tilde{l}$. He can buy coinsurance at a rate $\beta$ for a premium $(1+\lambda) \beta E \tilde{l}$, where $\lambda$ denotes the proportional loading factor. The agent chooses the coinsurance rate $\beta$ to maximize his intertemporal welfare

$$
W(\beta)=\max _{y_{\min } \leq y \leq y_{\max }} k v(y)+E U\left(z_{0}-(1-\beta) \tilde{l}-(1+\lambda) \beta E \tilde{l}, y\right) .
$$

This problem is equivalent to the portfolio allocation problem where full insurance, $\beta=1$, is equivalent to investing all wealth into the risk-free asset, $\alpha=0$. We therefore obtain the following result, which mirrors Proposition 8.

Proposition 10 If insurance is actuarially fair $(\lambda=0)$ then full coverage is optimal. If insurance is actuarially unfair $(\lambda>0)$ then partial coverage is optimal.

This is a direct consequence of Machina (1982), who has shown that most classical results in insurance are obtained in his Generalized Expected Utility model as long as the "local utility function" $M$ is concave in outcomes. In our special case, concavity of $M$ is implied by the concavity of $U(c, y)$ in $c$, see $(7)$.

Analogous to Proposition 9, we obtain the following comparative statics of the optimal insurance amount with respect to changes in $k$ and $\eta$.

Proposition 11 Suppose that $U(c, y)=u(c-\eta y)$ and $u$ is DARA.

1. The amount of insurance coverage is increasing in $k$.

2. The amount of insurance coverage is increasing in (decreasing in, independent of) $\eta$ if relative risk aversion is uniformly larger than (smaller than, equal to) unity.

If relative risk aversion is uniformly larger than one, individuals with anticipatory feelings and ex post disappointment buy more insurance compared to the case without disappointment aversion. This result is consistent with the observation that individuals have a preference for low deductibles - see e.g. Pashigian et al. (1966), Cohen and Einav (2007), Sydnor (2009). 


\section{Conclusion}

We proposed a new decision criterion under uncertainty by allowing individuals to extract utility from dreaming about the future and disutility from being disappointed ex post. Individuals then have an incentive to manipulate their beliefs about the future. We have described the mental process of how beliefs are formed to manage the trade-off between savoring and being disappointed. The preferences derived from this process are consistent with first-degree and second-degree stochastic dominance and might explain observed violations of the independence axiom, the equity premium puzzle, and the preference for low deductibles in insurance contracts. 


\section{References}

Akerlof, G.A., and W.T. Dickens, (1982), The economic consequences of cognitive dissonance, American Economic Review, 72, 307-319.

Ang, A., G. Bekaert, and J. Liu, (2005), Why stocks may disappoint, Journal of Financial Economics, 76, 471-508.

Bell, D.E., (1985), Disappointment in decision making under uncertainty, Operations Research, 33, 1-27.

Ben Mansour, S., E. Jouini, J.-M. Marin, C. Napp, and C. Robert, (2008), Are riskaverse agenst more optimistic? A Bayesian estimation approach, Journal of Applied Econometrics, 23, 843-860.

Brunnermeier, M.K., and J.A. Parker, (2005), Optimal expectations, American Economic Review, 95, 1092-1118.

Caplin, A.J., and J. Leahy, (2001), Psychological expected utility theory and anticipatory feelings, Quarterly Journal of Economics, 106, 55-80.

Carroll, P.J., Sweeny, K., and J.A. Shepperd, (2006), Forsaking optimism, Review of General Psychology, 10, 56-73.

Cohen, A., and L. Einav, (2007), Estimating risk preferences from deductible choice, American Economic Review, 97, 745-788.

Constandinides, G.M., (1990), Habit formation: A resolution of the equity premium puzzle, Journal of Political Economy, 98, 519-543.

Eeckhoudt L., B. Rey, and H. Schlesinger, (2007), A good sign for multivariate risk taking, Management Science, 53, 117-124.

Gollier, C., (1995), The comparative statics of changes in risk revisited, Journal of Economic Theory, 66, 522-536.

Gollier, C., (2005), Optimal illusions and decisions under risk, IDEI working paper 340, University of Toulouse.

Gul, F., (1991), A theory of disappointment aversion, Econometrica, 59, 667-686. 
Kimball, M.S., (1990), Precautionary savings in the small and in the large, Econometrica, 58, 53-73.

Kimball, M.S., (1993), Standard risk aversion, Econometrica, 61, 589-611.

Kopczuk, W., and J. Slemrod, (2005), Denial of death and economic behavior, Advances in Theoretical Economics, 5(1), Article 5. http://www. bepress/betje/advances/vol5/iss1/art5.

Kőszegi, B., and M. Rabin, (2006), A model of reference-dependent preferences, Quarterly Journal of Economics, 121, 1133-1165.

Kőszegi, B., and M. Rabin, (2007), Reference-dependent risk attitudes, American Economic Review, 97, 1047-1073.

Kreps, D.M., and E.L. Porteus, (1979), Temporal von Neumann-Morgenstern and induced preferences, Journal of Economic Theory, 20, 81-109.

Machina, M., (1982), Expected utility analysis without the independence axiom, Econometrica, 50, 277-323.

Machina, M., (1984), Temporal risk and the nature of induced preferences, Journal of Economic Theory, 33, 199-231.

Machina, M., (1987), Choice under uncertainty: Problems solved and unsolved, Journal of Economic Perspectives, 1, 281-296.

Machina, M.J., (1995), Non-Expected Utility and the Robustness of the Classical Insurance Paradigm, Geneva Papers on Risk and Insurance Theory, 20, 9-50.

McGraw, A.P., B.A. Mellers, and I. Ritov, (2004), The affective costs of overconfidence, Journal of Behavioral Decision Making, 17, 281-295.

Pashigian, B.P., L.L. Schkade, and G.H. Menefee, (1966), The selection of an optimal deductible for a given insurance policy, Journal of Business, 39, 35-44.

Rothschild, M., and J.E. Stiglitz, (1971), Increasing risk: II Its economic consequences, Journal of Economic Theory, 3, 66-84.

Shepperd, J.A., and J.K. McNulty, (1998), Mood following outcomes: The pivotal role of expectations, Working paper, University of Florida, Gainesville. 
Shepperd, J.A., and J.K. McNulty, (2002), The affective consequences of expected and unexpected outcomes, Psychological Science, 13, 85-88.

Shepperd, J.A., J.A. Ouelette, and J.K. Fernandez, (1996), Abandoning unrealistic optimism: Performance estimates and the temporal proximity of self-relevant feedback, Journal of Personality and Social Psychology, 70, 844-855.

Sydnor, J., (2009), (Over)insuring modest risks, Working paper, Case Western Reserve University.

Taylor, K.M., and J.A. Shepperd, (1998), Bracing for the worst: Severity, testing, and feedback timing as moderators of the optimistic bias, Personality and Social Psychology Bulletin, 24, 915-926.

Van Dijk, W.W., M. Zeelenberg, and J. van der Pligt, (2003), Blessed are those who expect nothing: Lowering expectations as a way of avoiding disappointment, Journal of Economic Psychology, 24, 505-516. 


\section{A Appendix: Proofs}

\section{A.1 Proof of Proposition 7}

Property 1 comes from the following sequence of inequalities:

$$
\eta E u^{\prime}\left(\widetilde{c}-\eta y^{*}\right) \geq \eta u^{\prime}\left(\mu-\eta y^{*}\right) \geq k(1-\eta) u^{\prime}\left(\mu-\eta y^{*}\right)
$$

Combining this with (10) implies that $(1-\eta) y^{*} \leq \mu-\eta y^{*}$, or equivalently, $y^{*} \leq \mu$. The second property is obtained by fully differentiating (10) with respect to $y^{*}$ and $\mu$, and by eliminating $k$. This yields

$$
\frac{d y^{*}}{d \mu}=\frac{-E u^{\prime \prime}\left(\widetilde{c}-\eta y^{*}\right)}{E u^{\prime}\left(\widetilde{c}-\eta y^{*}\right)}\left[(1-\eta) \frac{-u^{\prime \prime}\left((1-\eta) y^{*}\right)}{u^{\prime}\left((1-\eta) y^{*}\right)}+\eta \frac{-E u^{\prime \prime}\left(\widetilde{c}-\eta y^{*}\right)}{E u^{\prime}\left(\widetilde{c}-\eta y^{*}\right)}\right]^{-1} \geq 0
$$

It implies that $d y^{*} / d \mu$ is larger than unity if

$$
\frac{-E u^{\prime \prime}\left(\widetilde{c}-\eta y^{*}\right)}{E u^{\prime}\left(\widetilde{c}-\eta y^{*}\right)} \geq \frac{-u^{\prime \prime}\left((1-\eta) y^{*}\right)}{u^{\prime}\left((1-\eta) y^{*}\right)},
$$

where $y^{*}$ is such that $u^{\prime}\left((1-\eta) y^{*}\right)=E u^{\prime}\left(\widetilde{c}-\eta y^{*}\right)$. As shown by Kimball (1993), this is true if and only if $u$ is standard.

\section{A.2 Proof of Proposition 9}

Implicitly differentiating (10) with respect to $k, \eta$, and $\alpha$ for $\widetilde{c}=z_{0}+\alpha \widetilde{x}$ yields

$$
\begin{aligned}
y_{k}^{*} & =-\frac{(1-\eta) u^{\prime}\left((1-\eta) y^{*}\right)}{k(1-\eta)^{2} u^{\prime \prime}\left((1-\eta) y^{*}\right)+\eta^{2} E u^{\prime \prime}\left(z_{0}+\alpha \widetilde{x}-\eta y^{*}\right)}, \\
y_{\eta}^{*} & =\frac{k u^{\prime}\left((1-\eta) y^{*}\right)+\eta k(1-\eta) y^{*} u^{\prime \prime}\left((1-\eta) y^{*}\right)-\eta^{2} y^{*} E u^{\prime \prime}\left(z_{0}+\alpha \widetilde{x}-\eta y^{*}\right)}{\eta k(1-\eta)^{2} u^{\prime \prime}\left((1-\eta) y^{*}\right)+\eta^{3} E u^{\prime \prime}\left(z_{0}+\alpha \widetilde{x}-\eta y^{*}\right)}, \\
y_{\alpha}^{*} & =\frac{\eta E \widetilde{x} u^{\prime \prime}\left(z_{0}+\alpha \widetilde{x}-\eta y^{*}\right)}{k(1-\eta)^{2} u^{\prime \prime}\left((1-\eta) y^{*}\right)+\eta^{2} E u^{\prime \prime}\left(z_{0}+\alpha \widetilde{x}-\eta y^{*}\right)} .
\end{aligned}
$$

Note that $y_{k}^{*}>0$ and $y_{\alpha}^{*}<0$ at $\alpha=\alpha^{*}$ as $u$ DARA implies $E\left[\tilde{x} u^{\prime \prime}\left(z_{0}+\alpha^{*} \tilde{x}-\eta y^{*}\right)\right]>0$. Implicitly differentiating the first-order condition for $\alpha^{*}$,

$$
E \widetilde{x} u^{\prime}\left(z_{0}+\alpha^{*} \widetilde{x}-\eta y^{*}\right)=0,
$$


where $\alpha^{*}=\alpha^{*}(k, \eta)$ and $y^{*}=y^{*}\left(\alpha^{*}(k, \eta), k, \eta\right)$, with respect to $k$ implies

$$
\alpha_{k}^{*}=\frac{\eta y_{k}^{*} E \widetilde{x} u^{\prime \prime}\left(z_{0}+\alpha^{*} \widetilde{x}-\eta y^{*}\right)}{E \widetilde{x}^{2} u^{\prime \prime}\left(z_{0}+\alpha^{*} \widetilde{x}-\eta y^{*}\right)-\eta y_{\alpha}^{*} E \widetilde{x} u^{\prime \prime}\left(z_{0}+\alpha^{*} \widetilde{x}-\eta y^{*}\right)}
$$

The numerator is positive under $u$ DARA and the denominator can be written as

$$
\begin{aligned}
& E \widetilde{x}^{2} u^{\prime \prime}\left(z_{0}+\alpha^{*} \widetilde{x}-\eta y^{*}\right)-\eta y_{\alpha}^{*} E \widetilde{x} u^{\prime \prime}\left(z_{0}+\alpha^{*} \widetilde{x}-\eta y^{*}\right) \\
& =\frac{\left(\begin{array}{c}
k(1-\eta)^{2} u^{\prime \prime}\left((1-\eta) y^{*}\right) E \widetilde{x}^{2} u^{\prime \prime}\left(z_{0}+\alpha^{*} \widetilde{x}-\eta y^{*}\right) \\
+\eta^{2}\left(E u^{\prime \prime}\left(z_{0}+\alpha \widetilde{x}-\eta y^{*}\right) E \widetilde{x}^{2} u^{\prime \prime}\left(z_{0}+\alpha^{*} \widetilde{x}-\eta y^{*}\right)-\left(E \widetilde{x} u^{\prime \prime}\left(z_{0}+\alpha \widetilde{x}-\eta y^{*}\right)\right)^{2}\right)
\end{array}\right)}{k(1-\eta)^{2} u^{\prime \prime}\left((1-\eta) y^{*}\right)+\eta^{2} E u^{\prime \prime}\left(z_{0}+\alpha \widetilde{x}-\eta y^{*}\right)} \\
& <0 .
\end{aligned}
$$

The last inequality follows from the Cauchy-Schwartz inequality which implies $E u^{\prime \prime}\left(z_{0}+\alpha \widetilde{x}-\right.$ $\left.\eta y^{*}\right) E \widetilde{x}^{2} u^{\prime \prime}\left(z_{0}+\alpha^{*} \widetilde{x}-\eta y^{*}\right)-\left(E \widetilde{x} u^{\prime \prime}\left(z_{0}+\alpha \widetilde{x}-\eta y^{*}\right)\right)^{2}=E u^{\prime \prime}\left(z_{0}+\alpha \widetilde{x}-\eta y^{*}\right) E(\widetilde{x}-1)^{2} u^{\prime \prime}\left(z_{0}+\right.$ $\left.\alpha^{*} \widetilde{x}-\eta y^{*}\right)-\left(E(\widetilde{x}-1) u^{\prime \prime}\left(z_{0}+\alpha \widetilde{x}-\eta y^{*}\right)\right)^{2} \geq 0$. This proves $\alpha_{k}^{*}<0$. Implicitly differentiating (16) with respect to $\eta$ yields

$$
\alpha_{\eta}^{*}=\frac{\left(y^{*}+\eta y_{\eta}^{*}\right) E \widetilde{x} u^{\prime \prime}\left(z_{0}+\alpha^{*} \widetilde{x}-\eta y^{*}\right)}{E \widetilde{x}^{2} u^{\prime \prime}\left(z_{0}+\alpha^{*} \widetilde{x}-\eta y^{*}\right)-\eta y_{\alpha}^{*} E \widetilde{x} u^{\prime \prime}\left(z_{0}+\alpha^{*} \widetilde{x}-\eta y^{*}\right)}
$$

The denominator is negative as shown above. DARA implies $E \widetilde{x} u^{\prime \prime}\left(z_{0}+\alpha^{*} \widetilde{x}-\eta y^{*}\right)>0$ and thus $\operatorname{sign}\left(\alpha_{\eta}^{*}\right)=-\operatorname{sign}\left(y^{*}+\eta y_{\eta}^{*}\right)$. Furthermore

$$
y^{*}+\eta y_{\eta}^{*}=\frac{k u^{\prime}\left((1-\eta) y^{*}\right)\left(1-A_{r}\left((1-\eta) y^{*}\right)\right)}{k(1-\eta)^{2} u^{\prime \prime}\left((1-\eta) y^{*}\right)+\eta^{2} E u^{\prime \prime}\left(z_{0}+\alpha \widetilde{x}-\eta y^{*}\right)}
$$

where $A_{r}(z)=-z u^{\prime \prime}(z) / u^{\prime}(z)$. This implies $\alpha_{\eta}^{*}<(>,=) 0$ iff $A_{r}\left((1-\eta) y^{*}\right)>(<,=) 1$. 